\title{
Recent Issues of the Biochemistry and Molecular Biology of Trypanosoma cruzi
}

\author{
Luiz R Travassos/ ${ }^{+}$, Erney P Camargo
}

Disciplina de Biologia Celular, Universidade Federal de São Paulo and Departamento de Parasitologia, Universidade de São Paulo, 05508-900 São Paulo, SP Brasil

Key words: Trypanosoma cruzi - glycoconjugates - metacyclogenesis - gp-90 - gp-82 - phylogenesis

Recent issues of the biochemistry and molecular biology of Trypanosoma cruzi investigated by prestigious Brazilian research groups and discussed in the present meeting in commemoration of the 90th anniversary of the recognition of Chagas disease, center on glycoconjugates, their structure and function; differential gene expression in developmental stages of the parasite; organization and expression of multigene families encoding virulence factors; and the epidemiology, biochemistry and evolution of different strains of the parasite using rRNA sequences.

Almeida and coworkers have studied the complex structure of mucin-like glycoproteins from infective forms of $T$. cruzi and advanced in the knowledge of their immunological properties. These molecules are densely packed over the entire surface of trypomastigotes and contain O-glycosidically-linked oligosaccharides which are terminally substituted by numerous sialic acid units. Many of the biological properties of these GPIanchored mucins have been related to the presence of these acidic residues. Two major features of $T$. cruzi mucins emerged from studies by different groups: (a) in contrast with mammalian mucins, the O-linked oligosaccharide chains of $T$. cruzi mucins are bound via an internal unit of $\mathrm{N}$ acetylglucosamine; (b) sialic acid units are added to the oligosaccharide chains via a trans-sialidase reaction which uses sialylated donors from the medium. Mucins from epimastigotes and metacyclic forms differ from those of cell culture trypomastigotes in their apparent molecular mass, susceptibility to proteolysis and fine glycosidic structure. They also show peculiar characteristics in the lipidic moiety of the GPI anchors which are related with some of their immunological proper-

\footnotetext{
$\overline{{ }^{+} \text {Corresponding author. Fax: }}+55-11-571.5877$. E-mail: travassos.dmip@epm.br

Received 9 June 1999

Accepted 9 August 1999
}

ties. A striking feature of the infective trypomastigote mucins is the presence of terminal $\alpha$ galactopyranosyl units in their oligosaccharides which are targets of lytic antibodies isolated from patients with chronic Chagas disease. It is highly relevant that the successful chemotherapy in some chagasic patients can be monitored by the gradual decrease in the lytic antibody titers. These antibodies are now being detected by extremely sensitive chemiluminescent Elisa using very small amounts of purified trypomastigote mucins. Recent studies showed that trypomastigote mucins, but not those from epimastigotes or metacyclic forms potently induce the synthesis by stimulated macrophages of proinflammatory cytokines (IL12 and TNF- $\alpha$ ) and of nitric oxide. Since these effects are obtained using extremely reduced amounts of mucins they appear to have physiological relevance in vivo especially since mucins may be shed into the medium by $T$. cruzi infective forms. A most careful study is presently being carried out by Almeida et al. to correlate minimum structural requirements with immunobiological activity using powerful methods of purification and characterization of the mucins and their GPI moieties. The precise role of these components of T. cruzi in the pathophysiology of experimental and human infection by this parasite may thus soon be unrevealed.

Metacyclogenesis in $T$. cruzi is the central project of Goldenberg and coworkers and it has been addressed using differential gene expression. A chemically defined medium was developed that mimetizes the triatomine urine so that metacyclic forms obtained from either of them show comparable biological properties.To isolate and characterize the genes specifically expressed in the terminal stages of differentiation as well as during the process of metacyclogenesis, a technique called "representation of differential expression" (RDE) has been developed. The method is based on the amplification by PCR of specific gene sequences isolated by subtractive hybridization, using oligonucleotides representing the mini-exon and polyA sequences as primers for the PCR reaction, thus 
allowing amplification of entire mRNA sequences of the parasite. By using RDE during the metacyclogenesis process (different times of differentiation), several genes specifically expressed were isolated. Some of these genes are transitionally expressed in the differetiating cells but not in epi- or trypomastigotes. Others are continuosly expressed in differentiating cells and in metacyclics but not in epimastigotes. Sequenced genes showed no homology with published sequences and their regulation is post-transcriptional. The authors plan to characterize these genes in terms of biological activity as well as to sequence other genes isolated using the same methodology. These studies are aimed not only at a better understanding of the metacyclogenesis process but also at providing new tools for study of the modulation of gene expression in T. cruzi.

Franco da Silveira and collaborators have studied the genomic organization and expression of genes encoding two glycoproteins, gp90 and gp82. Both gp90 and gp82 are involved in the penetration of the parasite into host cells. The gp82 can induce a $\mathrm{Ca}^{2+}$ signal in target cells, an event which is essential for T. cruzi internalization. Immunization with the gp 90 or gp 82 can protect mice against challenge by a virulent strain of $T$. cruzi. Structurally, both the gp82 and gp90 are surface antigens containing $\mathrm{N}$-linked oligosaccharide chains and anchored to the membrane via a GPI anchor. The cDNA clones encoding both antigens were isolated from expression libraries using monoclonal antibodies. Several post-translational modifications such as N-glycosylation and addition of a GPI anchor were observed. The ORF of the gp 82 cDNA clones encodes polypeptides of 516 to 595 amino acids. Sequence analysis of gp 90 and gp 82 showed 40-60\% identity at amino acid level with $T$. cruzi gp85/sialidase family. The genes $g p 90$ and $g p 82$ are organized in subsets spread in the genome. Using a $T$. cruzi YAC library it was shown that these genes and/or $g p 85$ are linked at multiple sites of the genome. All of these related genes may be linked in different chromosomal loci while some sub-telomeric regions contain sequences associated with the gp90 and gp85 genes. Northern and Western blotting showed that $g p 90$ and $g p 82$ are preferentially transcribed and expressed in metacyclic forms, but by using other methods (e.g. RNAPCR) the presence of gp 82 and gp90 transcripts were also found in epimastigotes and blood trypomastigotes. Homologues of $g p 82$ and $g p 90$ and of gp 85 have been identified also in $T$. rangeli suggesting that these genes were originated from a common ancestral gene present in several members of Trypanosoma genus.
A challenge to researchers on Chagas disease has been the correlation of molecular markers of the parasite with the epidemiology of this infection. In fact, $T$. cruzi has a remarkable genetic heterogeneity as evidenced by isoenzymatic phenotypes, kinetoplast DNA fragments, DNA finger-prints and molecular karyotype. Using these markers the various strains of the parasite could not be grouped in few clusters. On the contrary, a clear dichotomy in the $T$. cruzi taxon was observed using other genetic markers such as ribosomic RNA. Zingales and coworkers have shown that a sequence of the D7 domain of the rRNA $24 \mathrm{~S}$ alpha (LSU) has a clear dimorphism in $T$. cruzi strains, allowing definition of two groups which also present dimorphism in the gene spacer of the mini-exon. RAPD analysis showed a genetic variability among isolates but the resulting dendogram evidenced two main branches which correlate with groups defined according to the rDNA and mini-exon analysis. These branches have been designated as Lineage 1 and Lineage 2 (L1 and L2) of T. cruzi.

The ribosomic cistron has structural variations in isolates from both lineages. Riboprinting and restriction profiles of the transcribed internal spacer gave rise to dendograms which group the isolates in the two previously defined lineages. The sequences of L1 and L2 promoters showed a similarity of $82 \%$ whereas the sequence identity within the same lineage was of $98 \%$. Such degree of homology further supports the division of $T$. cruzi in two groups. The activity of the ribosomic promoters was investigated by the transitory expression of a reporter gene. The authors concluded that the L1 promoters are only expressed in L1 strains whereas L2 promoters are expressed in representative isolates of both lineages.

Phylogenetic reconstructions based on the rRNA 18S (SSU) gene show that the divergence between both $T$. cruzi lineages is greater than the distance separating four Leishmania species. The time of divergence between L1 and L2 is estimated in 88 million years confirming their status as distinct units in evolution.

The importance of lineages L1 and L2 in the epidemiology of Chagas disease was investigated in 200 isolates of T. cruzi from 12 Brazilian states based on PCR typing of the LSU gene and of the mini-exon. Data show a strong association of L1 with the domestic cycle of the parasite with greater parasitemia in inhabitants of endemic areas, whereas L2 predominated in the sylvatic cycle. L2 has also been isolated from human cases of trypanosomiasis in the Amazon region where T. cruzi is enzootic. The adaptation of L1 to primates and of L2 to marsupials has been confirmed from studies carried out in the Atlantic Forest in Rio de Janeiro. 
These results indicate that $T$. cruzi can be divided in two lineages at the genotypic and physiological levels. It is important to characterize the sub-groups within each lineage aiming at a better understanding of the epidemiology and of the complexity of the clinical manifestations in Chagas disease.
All four communications by outstanding research groups in Brazil centering on the biochemistry and molecular biology of T. cruzi are good representatives of a scientific investigation using advanced methodology to understand the biology of $T$. cruzi and the pathophysiology of Chagas disease. 